\title{
Cellular Scanning Strategy for Selective Laser Melting: Capturing Thermal Trends with a Low-Fidelity, Pseudo-Analytical Model
}

\author{
Sankhya Mohanty and Jesper Hattel \\ Department of Mechanical Engineering, Building 425, Produktionstorvet, Technical University of Denmark (DTU), \\ 2800 Lyngby, Denmark \\ Correspondence should be addressed to Sankhya Mohanty; samoh@mek.dtu.dk
}

Received 28 February 2014; Revised 20 May 2014; Accepted 21 May 2014; Published 18 June 2014

Academic Editor: Changzhi Wu

Copyright (C) 2014 S. Mohanty and J. Hattel. This is an open access article distributed under the Creative Commons Attribution License, which permits unrestricted use, distribution, and reproduction in any medium, provided the original work is properly cited.

\begin{abstract}
Simulations of additive manufacturing processes are known to be computationally expensive. The resulting large runtimes prohibit their application in secondary analysis requiring several complete simulations such as optimization studies, and sensitivity analysis. In this paper, a low-fidelity pseudo-analytical model has been introduced to enable such secondary analysis. The model has been able to mimic a finite element model and was able to capture the thermal trends associated with the process. The model has been validated and subsequently applied in a small optimization case study. The pseudo-analytical modelling technique is established as a fast tool for primary modelling investigations.
\end{abstract}

\section{Introduction}

Selective laser melting (SLM) is a rapid manufacturing process which involves active localized heating and melting of metal powder to form components. The transient temperature field/distribution during the process has a major role in the process and has been observed across literature to have a direct implication on the distortion and residual stresses in final products. Thus, thermal analysis of the process continues to be the most active field of research with regard to modelling of selective laser melting [1].

A popular approach to analysis of manufacturing processes involving flow is through computational fluid dynamics (CFD). This is a standard method in the field of welding simulations and has, thus, also found its applications in modelling SLM. However, the transiency and specifications of the process limit the size of the domain that can be modelled using a CFD approach. Thus, CFD techniques are usually limited to simulation of single or few melting tracks [2-4].

A second strategy is to approach the problem using techniques of computational solid mechanics (CSM). Using certain methods, CSM calculations replace the convective melt-dynamics with equivalent conductive heat transfer. This simplifies the simulation and, thereby, allows inclusion of more physical phenomena into the model as well as a larger size of modelled domain. Thus, several works involving $2 \mathrm{D}$ or $3 \mathrm{D}$ thermal analysis of the process utilize this approach $[5,6]$.

The increasing availability of computational resources over the last decades has fueled the adoption of these methods as standard choices of numerical modelling across academia and industry. Although CFD and CSM have very different approaches, both these methods are based on rigorous mathematical concepts and have been the subject of several numerical experiments and analyses since their conception.

Consequently, there has been increased propensity towards coupled thermofluid dynamic or coupled thermomechanical modelling of manufacturing processes. While this has led to more accurate predictions and better understanding of the process, it has also led to its own nonusability. Both the numerical modelling techniques are riddled with extensive computational resource and time requirements. The review article on thermal analysis methods in selective laser melting by Zeng et al. [1] also tells the same story. These large time requirements inhibit the applicability of the models for any secondary analysis such as process optimization, uncertainty analysis, and factor analysis. 
To tackle the problem of large model evaluation time, several mathematical approaches have been proposed, collectively known as surrogate modelling techniques [7]. These techniques rely on creation of response surfaces and/or emulators that mimic the behavior of the simulation model and aim to substitute the model evaluations by evaluations of response surface. However, these response surfaces are not universal for each manufacturing process, and thus each significantly different set of process parameters would require creation of new response surfaces. Considering the minimum number of model evaluations required to construct the response surface and inherent transiency of the process, this technique is inapplicable towards optimization of selective laser melting.

Instead, in this paper, a fast, low-fidelity modelling technique is introduced which can be utilized for optimization of the selective laser melting and most thermally driven additive manufacturing processes. The technique draws upon several existing modelling concepts and tries to combine them in an innovative manner. The model is developed to serve a complementary role in process optimization by quick primary evaluations rather than as a complete substitute for accurate numerical modelling.

\section{Pseudo-Analytical Model}

2.1. Governing Equations. The governing equation for a thermal diffusion driven heat transfer can be given as

$$
\begin{aligned}
\rho C_{p} \frac{\partial T}{\partial t}= & \frac{\partial}{\partial x}\left(k_{x x} \frac{\partial T}{\partial x}\right)+\frac{\partial}{\partial y}\left(k_{y y} \frac{\partial T}{\partial y}\right) \\
& +\frac{\partial}{\partial z}\left(k_{z z} \frac{\partial T}{\partial z}\right)+\dddot{\Phi}
\end{aligned}
$$

where $T$ is the temperature, $\rho$ is the density of the material, $C_{p}$ is the specific heat, $k_{x x}, k_{y y}$, and $k_{z z}$ are the conductivity along the $x, y$, and $z$ direction, respectively, and $\dddot{\Phi}$ represents the volumetric heat source. In the simple case of nontemperature dependent homogeneous material properties, the heat transfer equation reduces to

$$
\rho C_{p} \frac{\partial T}{\partial t}=k \nabla^{2} T+\dddot{\Phi}
$$

The above equation states that the rate of change of energy of a body is equal to the sum of energy added to the body through an external heat source and the energy conducted into the body across its boundaries. Thus, for the simple linear case, the total change in temperature of the body can be assumed to be the cumulative temperature change due to two independent phenomena, namely, the heat addition by the external source and the conductive heat transfer; that is,

$$
\begin{aligned}
\frac{\partial T^{*}}{\partial t} & =\frac{\dddot{\Phi}}{\rho C_{p}}, \\
\frac{\partial T}{\partial t} & =\frac{k}{\rho C_{p}} \nabla^{2} T^{*}
\end{aligned}
$$

It is necessary to mention that the above splitting of equations is not accurate in the general case of material nonlinearity but is valid for the simplest case as stated. The equations are solved using a time-advancing algorithm; that is, the modelling time domain is divided into several discrete time steps and the pseudo-analytical solution is restarted at the beginning of each time step. To achieve the pseudoanalytical solution, several techniques and approximation strategies are applied, which are described in the following sections.

2.2. Discontinuous Modelling. In numerical modelling using finite element or finite volume methods, it is a standard practice to model the problem domain by dividing it into a set of nodes/control volumes and therefrom developing a system of algebraic equations which can be solved easily. The procedure involves discretization of the partial differential equation describing the heat transfer and relies on the convergence of errors with increasing number of nodes/control volumes. The discontinuous finite element method further allows interelement discontinuities and uses stabilizing terms and numerical fluxes to ensure continuity of solution variables.

A similar approach has been undertaken while developing the pseudo-analytical model. The problem domain is divided into several smaller domains resembling a structured mesh of control volumes. The solution variable (which is the temperature field in this case) in each of the small domain or element is weakly coupled with the values in adjacent domains through numerical fluxes at the boundary. Thus, instead of solving a system of equations with global boundary conditions, the heat transfer equation is solved for each smaller domain with equivalent interdomain boundary conditions. More specifically, for the current 2.5D pseudoanalytical model, each of the domains experiences an equivalent boundary temperature $\left(T^{\text {cond }}\right)$ which is an average of the temperatures of the adjacent domains

$$
T^{\mathrm{cond}}=\frac{1}{j} \sum_{i=1}^{j}\left(T_{i}\right)
$$

where $j$ is the number of adjacent domains and $T_{i}$ is the temperature in the $i$ th adjacent domain of the domain whose equivalent boundary temperature is being calculated.

The discontinuous domains are also used for calculation of time step size for the time-advancing algorithm by choosing the time step equal to the time required by the center of the laser beam to move from one domain to the center of the adjacent domain.

2.3. Laser Beam as Heat Source. The moving laser beam is assumed to have a Gaussian power distribution. The beam is modelled on a smaller grid corresponding to the area containing $>95 \%$ of the beam power, both at the start and end of a time step. The beam is converted into an equivalent body 


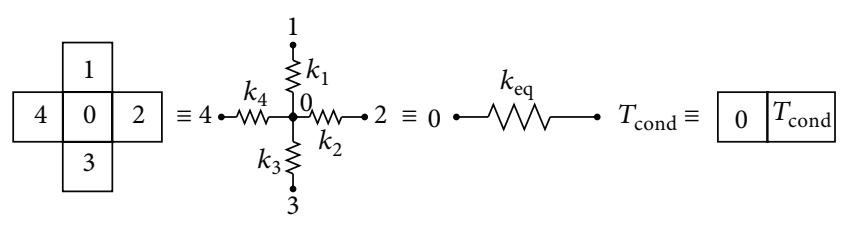

FIGURE 1: Defining $T_{\text {cond }}$ using a lumped capacitance thermal circuit.

heat source by integrating the energy delivered by the moving beam during one time step

$$
\begin{aligned}
\dddot{\Phi}=( & \left(2 \alpha P_{0} / \pi \omega_{0}^{2}\right) \\
& \left.\times \iiint e^{-\left[2\left\{\left(x-x_{0}-v_{x} t\right)^{2}+\left(y-y_{0}-v_{y} t\right)^{2}\right\} / \omega_{0}^{2}\right]} d x d y d t\right) \times\left(t_{\text {step }}\right)^{-1},
\end{aligned}
$$

where $P_{0}$ is the power of the laser beam, $\omega_{0}$ is the beam $1 / e^{2}$ radius and $\alpha$ is the absorptivity of the laser beam in the material, $\left(v_{x}, v_{y}\right)$ is the velocity of the laser beam in the $x$ - and $y$-direction, $\left(x_{0}, y_{0}\right)$ are the initial location of laser beam, and $t_{\text {step }}$ is the time step size.

The smaller grid representing the laser beam is then appropriately moved across the global domain based on the scanning strategy being simulated. In the current model, the effects of angle of incidence of the beam are neglected for simplification.

\subsection{Lumped Capacitance and Newtonian Cooling. As a sim-} plification of the transient heat transfer, the discontinuous domains are treated as lumped capacitances at the start of each intermediate step during the global time step. This allows easier calculations of $T^{\text {cond }}$ and application of analytical solutions within each discontinuous domain. With such an assumption, Newton's law of cooling can be used to model scenarios involving external convective cooling. The convective cooling is again treated as an independent phenomenon and therefore is to be applied using the same procedure as for conductive heat transfer (discussed below).

2.5. Conductive Heat Transfer. The solution of (4), obtained through the splitting of the heat transfer equation, would provide temperature due to conductive heat transfer inside the domain. In each of the discontinuous domains, the four boundaries are symmetric with respect to the center of the domain where the temperature is calculated. Therefore, the scenario is assumed to be similar to $1 \mathrm{D}$ heat conduction in a semi-infinite body with a surface temperatur $T^{\text {cond }}$ and an equivalent conductivity $k_{\mathrm{eq}}$ (see Figure 1). It is further assumed that the multiple boundaries have a cumulative effect on the temperature at the center of the domain. The analytical solution for the temperature at the center of the discontinuous domain could then be given by

$$
T(x, t)=T^{\text {cond }}+\left(T_{i}-T^{\mathrm{cond}}\right) \cdot \operatorname{erf}\left(\frac{x \sqrt{\rho C_{p}}}{\sqrt{4 k_{\mathrm{eq}} t}}\right),
$$

TABLE 1: Parameters for model validation.

\begin{tabular}{lc}
\hline Density & $4200 \mathrm{~kg} / \mathrm{m}^{3}$ \\
Specific heat & $821 \mathrm{~J} /(\mathrm{kg}-\mathrm{K})$ \\
Thermal conductivity & $27 \mathrm{~W} /(\mathrm{m}-\mathrm{K})$ \\
Solidus temperature & $1600^{\circ} \mathrm{C}$ \\
Liquidus temperature & $1650^{\circ} \mathrm{C}$ \\
Boiling temperature & $3201^{\circ} \mathrm{C}$ \\
Laser power & $120 \mathrm{Watt}$ \\
Laser beam diameter & $70 \mu \mathrm{m}$ \\
Laser power distribution profile & $\mathrm{Gaussian}$ \\
Scanning speed & $800 \mathrm{~mm} / \mathrm{s}$ \\
Environment temperature & $2^{\circ} \mathrm{C}(275 \mathrm{~K})$ \\
\hline
\end{tabular}

where $x$ is the distance between the center of the discontinuous domain and its boundary, $t$ is the time, and $T_{i}$ is the temperature at the starting of the conductive heat transfer.

In the current model, the conductive heat transfer is assumed to occur in two stages. In the first stage, corresponding to first half of the time step, the temperature field is calculated based on the temperatures obtained at the end of the previous time step. Then, the volumetric heat source is added and the temperature is recalculated. The second stage of conductive heat transfer then follows with the assumption that the temperature in each of the domains has again become constant.

\section{Validation of Model}

For validating the pseudo-analytical model with respect to a high fidelity finite element model, two test simulations were set up. Both the test cases used the same physical domain, differing only in the scanning strategy, that is, the movement pattern of the laser beam. The physical domain used for simulation was a $500 \mu \mathrm{m} \times 500 \mu \mathrm{m} \times 50 \mu \mathrm{m}$ block having constant material properties given in Table 1 . The two scanning strategies being simulated were out-spiral and parallel scanning strategies (Figures 2(b) and 2(c), resp.). The processing parameters used in both test cases are also tabulated in Table 1. While both the finite element model and the pseudo-analytical model were used to simulate the same test cases, the physical domain was handled differently in each model, as discussed below.

\subsection{Model Domain Description}

3.1.1. 3D Finite Element Model. The 3D FE model was implemented using the commercial software ABAQUS. The standard 8-noded heat transfer element DC3D8 was chosen for developing the model. A structured mesh of $50 \times 50 \times 1$ elements was generated with an element edge length of $10 \mu \mathrm{m}$ along the $x y$ plane and $50 \mu \mathrm{m}$ along the $z$-direction. The mesh dimensions were chosen based on a mesh sensitivity analysis. The domain along with the mesh is shown in Figure 3.

User-defined subroutines were utilized for defining the laser beam as a body heat flux and for defining the material 


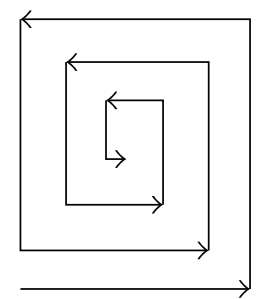

(a)

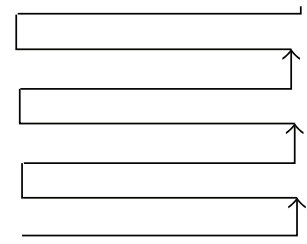

(d)

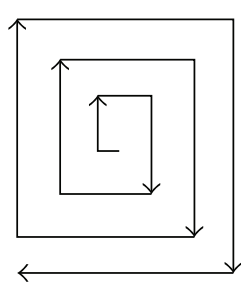

(b)

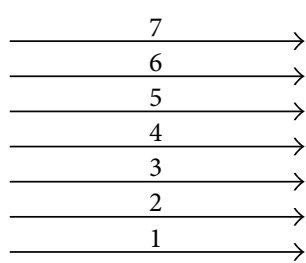

(c)

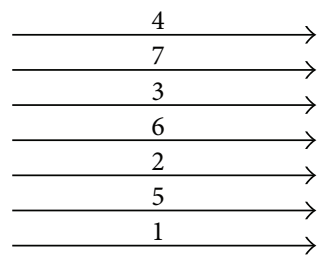

(e)

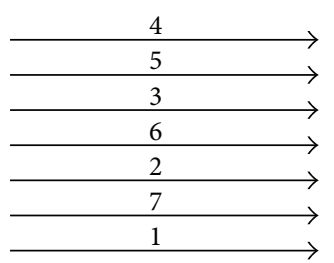

(f)

FIGURE 2: Scanning strategies for selective laser melting (a) In-spiral, (b) out-spiral, (c) parallel, (d) antiparallel, (e) parallel-interlaced, and (f) parallel-interlaced-reverse.

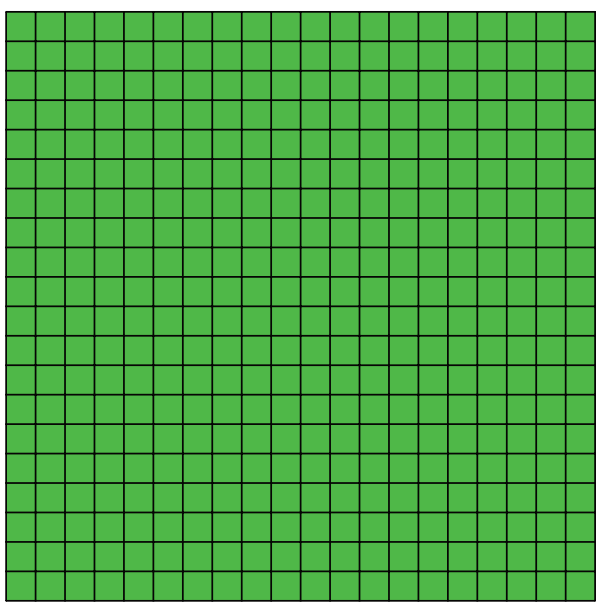

(a)

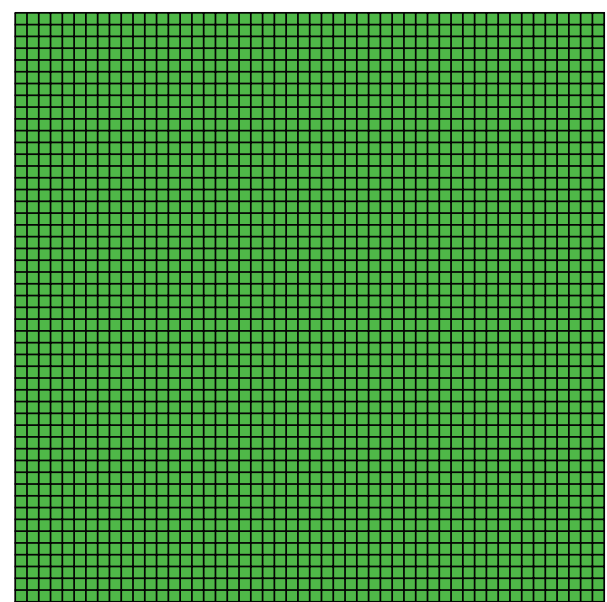

(b)

FIGURE 3: Structured mesh on the problem domain for model validation: coarse mesh (a) and a fine mesh obtained after mesh convergence study (b).

properties and field variables. No additional boundary conditions were applied on the domain, thereby ensuring a default adiabatic boundary. The model was simulated using a direct solver with an adaptive time stepping method limiting the maximum rise of temperature in each time step to $100^{\circ} \mathrm{C}$.

For further comparison, a second implementation of the 3 D FE model with a coarse structured mesh of $20 \times 20 \times 1$ elements was carried out. The time step size was chosen to be the same as the one calculated for the pseudo-analytical method so as to ensure minimum differences between the two models (i.e., the ABAQUS and the pseudo-analytical model).

3.1.2. 2.5D Pseudo-Analytical Model. In the pseudo-analytical model, a coarse meshing strategy was used. The mesh divided the physical domain into $20 \times 20 \times 1$ smaller domains of edge dimensions $25 \mu \mathrm{m} \times 25 \mu \mathrm{m} \times 50 \mu \mathrm{m}$ with two additional ghost domains along the $x$ - and $y$-directions for applying boundary conditions $(22 \times 22 \times 1$ domains in total $)$. A different meshing strategy was used so as to provide a comparative analysis of the capabilities of the pseudo-analytical model relative to the ABAQUS based FE model. Due to the larger mesh dimensions in the pseudo-analytical model, the time step size was also larger as compared to the fine 3D FE model.

3.2. Selection of Comparison Criteria. In the SLM process, it is desirable to have lower maximum temperatures and a greater thermal homogeneity while ensuring adequate melting and coalesced resolidification of the entire physical domain. Lower maximal temperatures during processing would ensure a smaller melt pool and reduce issues such as "balling effect" and segregation in case of alloys. Higher thermal homogeneity, that is, lower thermal gradients would 


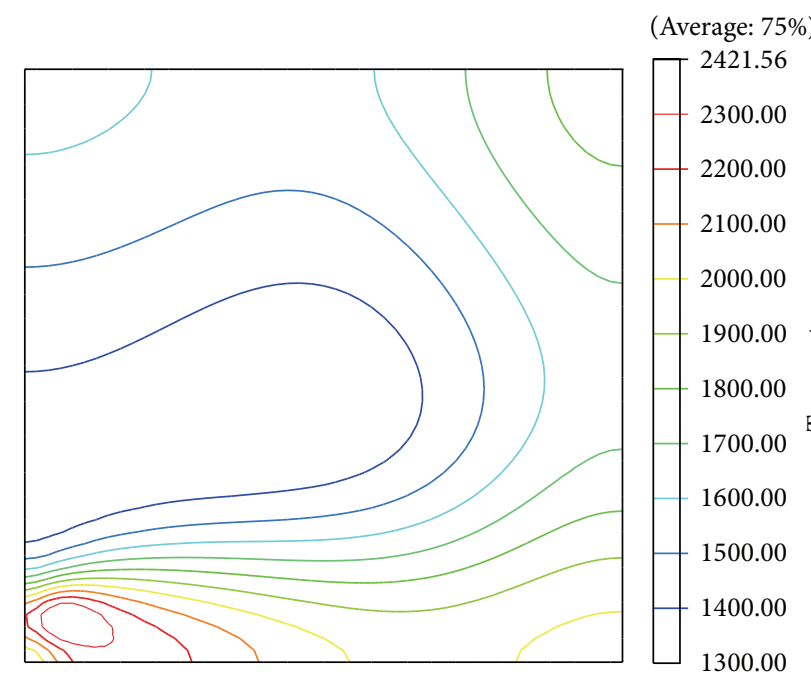

(a)

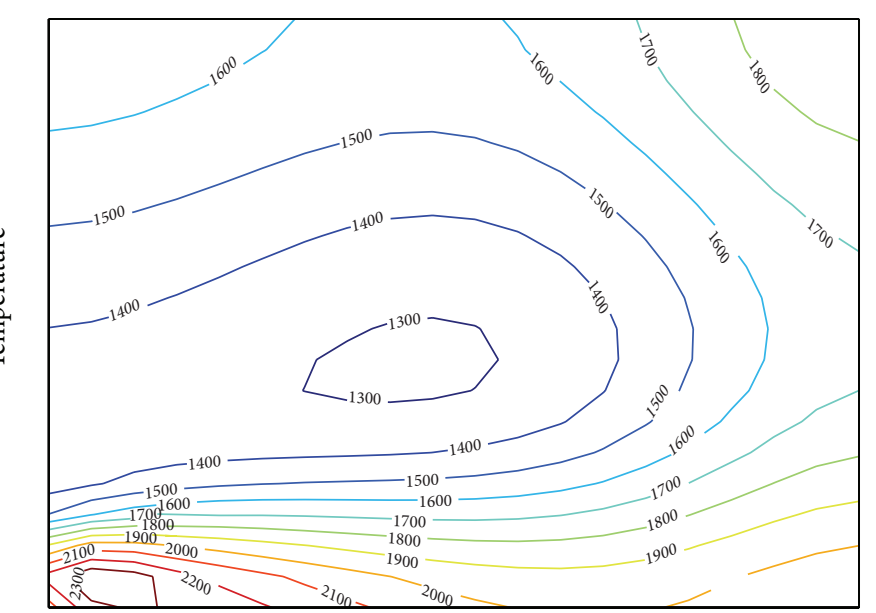

(b)

(Average: $75 \%$ )

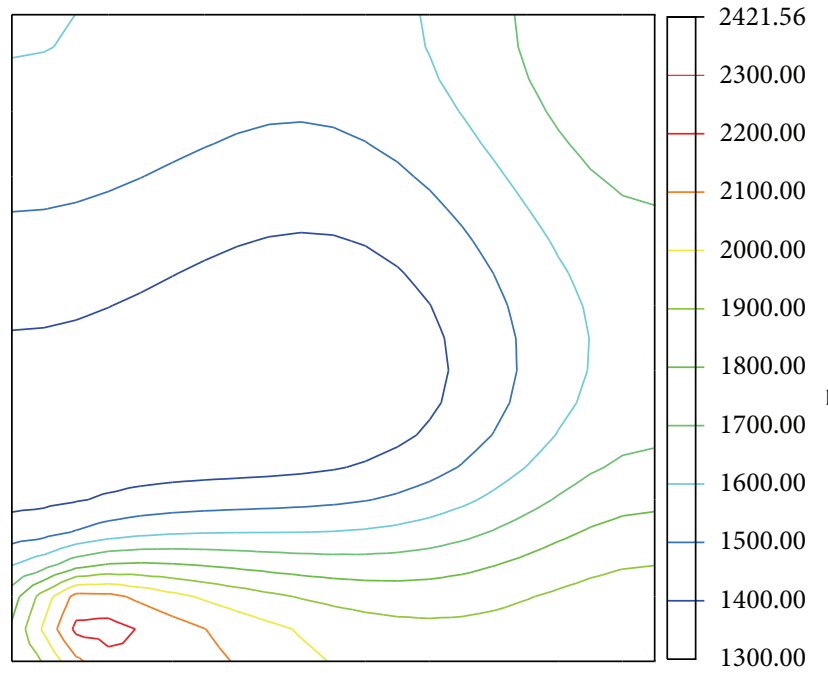

(c)

FIGURE 4: Temperature profile with 3D FE model (a) and pseudo-analytical model on a coarse mesh (b) and coarse 3D FE model (c) for out-spiral scanning strategy.

result in lower deformations and residual stresses, which are known issues with SLM. Therefore, the maximum temperature and thermal homogeneity were selected as criteria for comparison of the predictive capability of the pseudoanalytical model with respect to the more accurate finite element model. The maximum temperature is represented by $T_{\max }$, while the thermal homogeneity was quantified using the maximum standard deviation of the temperature field during the process and is represented by $\sigma_{\text {temp }}^{\max }$.

A further issue with SLM is the density of the products created by the process. Macroporosities are a recurring feature created due to inadequate melting and coalescence by resolidification. Thus, it is desirable to track the melting and resolidification of the domain during numerical modelling of SLM. Therefore two criteria, namely, molten zones and overheated zones, were introduced to compare the models. A dimensionless number $\theta$ was defined by combining the four criteria as a single indicator value of the efficacy of the process being simulated

$$
\theta=\left(\frac{T_{\max }}{\sigma_{\text {temp }}^{\max }}\right) * \frac{(1+V)}{1+M}
$$

where $T_{\max }$ is the maximum temperature during the simulation, $\sigma_{\text {temp }}^{\max }$ is the maximum value of standard deviation of temperature during simulation, and $V \& M$ are the number of overheated zones and number of molten zones at the end 


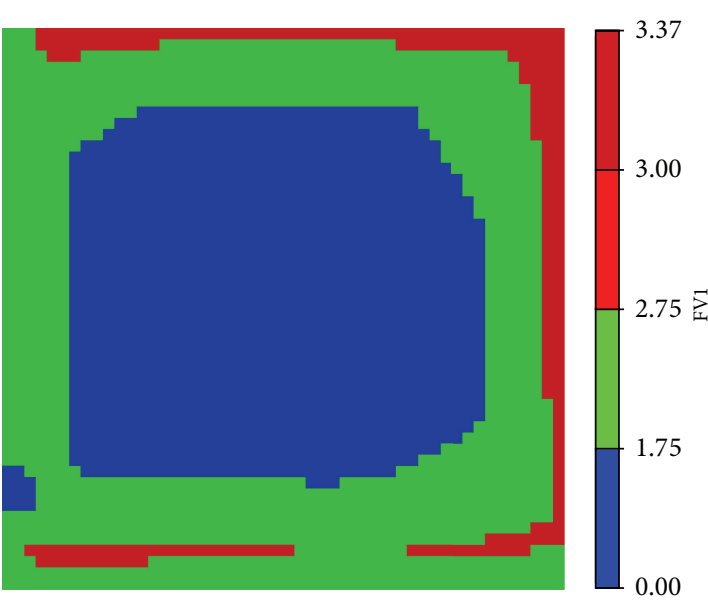

(a)

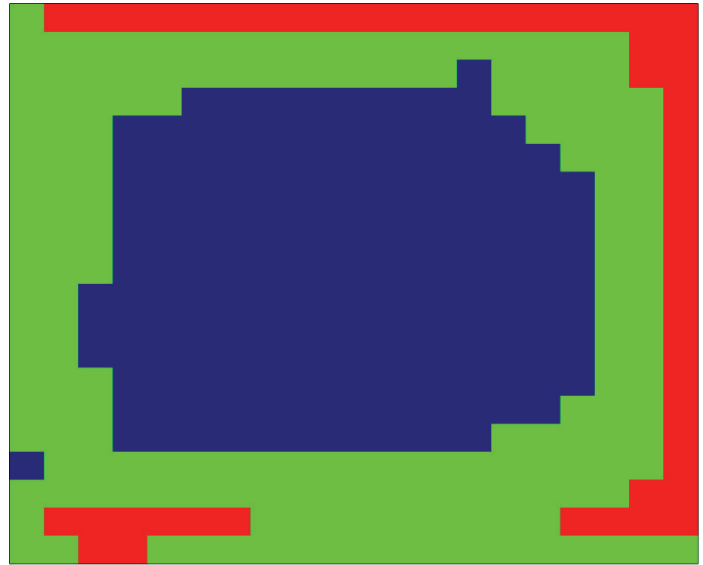

(b)

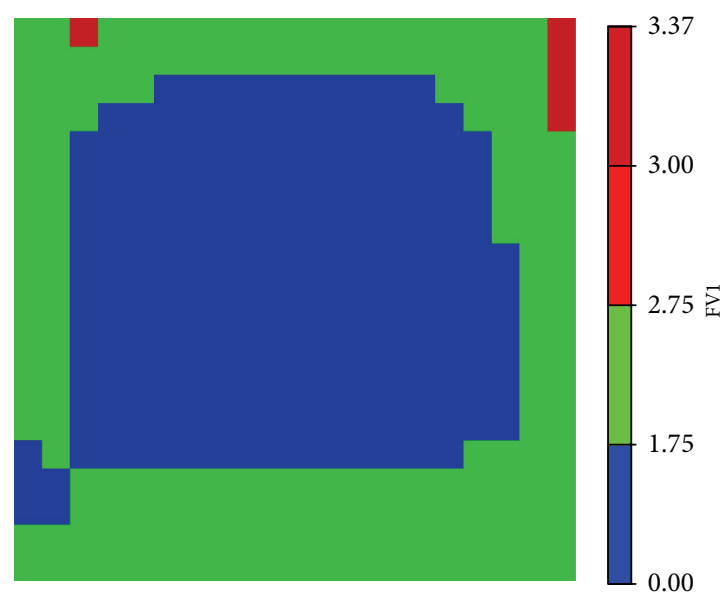

(c)

FIGURE 5: State of zones with 3D FE model (a) and pseudo-analytical model on a coarse mesh (b) and coarse 3D FE model (c) for out-spiral scanning strategy (nonmolten (blue), molten (green), and overheated (red)).

of simulation, respectively, defined based on the temperature history of each smaller domain as

Temperature $>$ Liquidus Temperature $\equiv$ Molten Zone,

Temperature

$>\frac{\text { (Liquidus Temperature }+ \text { Boiling Temperature) }}{2}$

$\equiv$ Overheated Zone.

3.3. Out-Spiral Scanning Strategy. Figure 2(b) shows the pattern of movement of the centre of the laser beam on the domain for the out-spiral scan path. In this scanning strategy, the laser starts near the center of the physical domain and moves away towards the edges following a spiral path. The out-spiral scan strategy is designed such that there is a minimum distance of $50 \mu \mathrm{m}$ between any two parallel tracks. The entire scan path is traversed by the laser beam in $6.09375 * 10^{-3}$ seconds without any discontinuity.
The temperature distribution obtained at the end of the scan is shown in Figure 4 for both the models. It can be observed that the results are quite similar, especially in the region under the laser beam. In this region, the temperature field is dominated by the external heat flux as compared to the flux due to the temperature gradients. As the major source of difference between the two models lies in the manner the conductive heat transfer is handled, the similarity in the temperature distribution in this region is as expected. In regions far away from the laser beam, the results between the two models are similar as more of the conditions for accurate applicability of analytical solutions start to become justified. The difference in the temperature field prediction is therefore only pronounced in the vicinity of the laser beam. It is also apparent from the plots that the pseudo-analytical method predicts lower temperature values compared to the $3 \mathrm{D} F E$ model. This is an outcome of the assumption of each discrete domain as a semi-infinite body, which results in a calculated temperature at each new time step that is closer to the initial temperature of the domain at the beginning of the time step. 


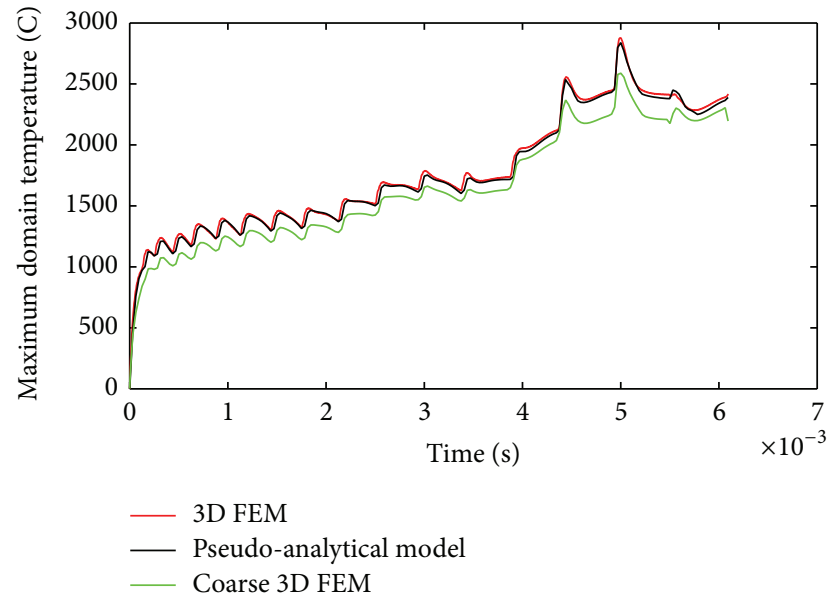

Figure 6: History plot for maximum temperature in modelled domain for out-spiral scan path.

Another result of importance during simulations of selective laser melting, as discussed earlier, is tracking the melting and consolidation. The characterization of each domain/element as powder, molten, or overheated zone is achieved by using Heaviside functions of the temperature, and is thus influenced by the accuracy of prediction of temperature. Figure 5 shows the results of the zoning for both the models at the end of the scan path.

While the temperature fields predicted by the two models look similar, differences in the zoning results are apparent. Thus, for proper validation it is necessary to also consider the temporal behavior of the models. The maximum temperature in the entire problem domain is tracked throughout the scan and is shown in Figure 6. It can be observed that the maximum temperature predictions in the pseudo-analytical model are lower than the 3D FE model yet follow the same trends. The maximal temperature predictions from the two models show incoherence at instances when either the laser beam changes the direction or while it is near the boundary of the problem domain. The pseudo-analytical model is unable to properly predict the temperatures at the boundaries as the assumption of symmetry inside each discrete domain breaks down. However, this issue is mitigated by adding additional ghost elements at the boundary or simply considering a slightly larger domain while modelling (in practice, selective laser melting uses a much larger powder layer than the component size). In case of change of direction, the assumption of an averaged boundary temperature for each discrete domain in the pseudo-analytical model leads to a lower temperature in the domain. However, in both these cases, the total amount of heat in the system is still conserved but is distributed differently. This argument is validated by plotting the average temperature of the whole domain throughout the scan path for both the models, as shown in Figure 7. Figure 8 shows that the standard deviations of temperature in the two models are not exactly the same (thereby compensating for lower maximal temperatures while having same average temperature) but follow similar trends.

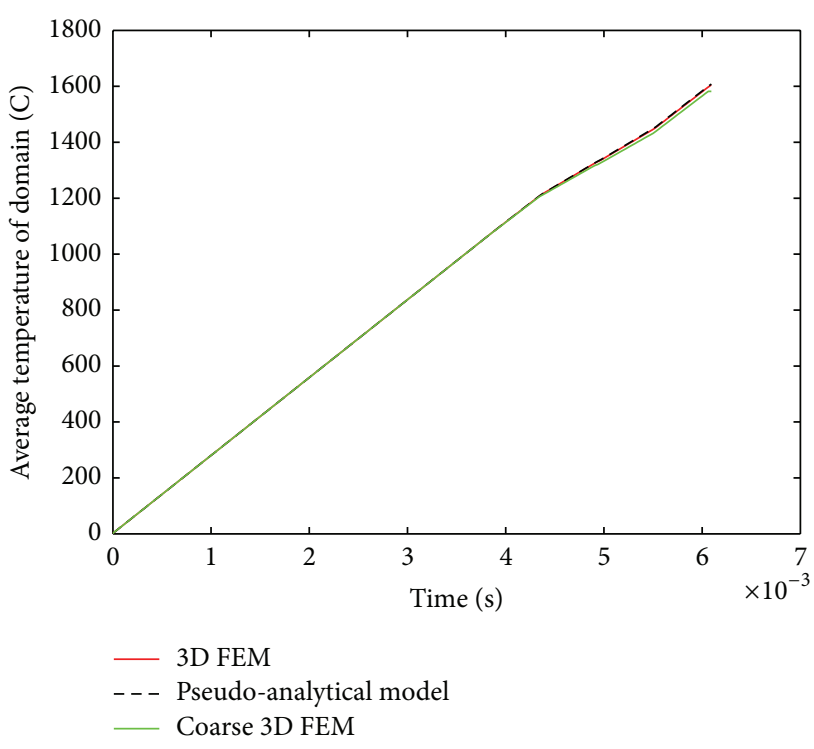

Figure 7: History plot for average domain temperature for outspiral scan path.

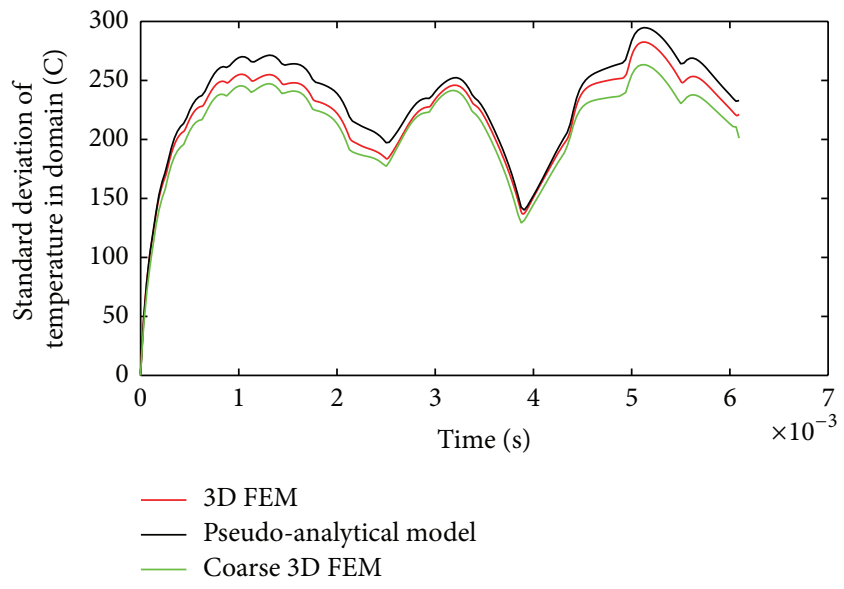

FIGURE 8: History plot for standard deviation of temperature in modelled domain.

3.4. Parallel Scanning Strategy. The parallel scan path (Figure 2(c)) is the most popular and used scan strategy for SLM. In this scanning strategy, parallel long unidirectional tracks are traced with a fixed distance of $50 \mu \mathrm{m}$ between adjacent tracks. The entire scan path is traversed in $5.9375 *$ $10^{-3}$ seconds with several discontinuous jumps of the laser beam from the end of one track to the beginning of the next track. It has been assumed that the maximum speed of laser beam is much greater than the scan speed being simulated, thereby making the time for shifting the laser beam to the beginning of the next track negligible.

Figure 9 shows the temperature distribution at the end of the parallel scanning strategy obtained using the three models. As in the case of out-spiral scanning strategy, the pseudo-analytical method is able to predict the temperature 
(Average: $75 \%$ )

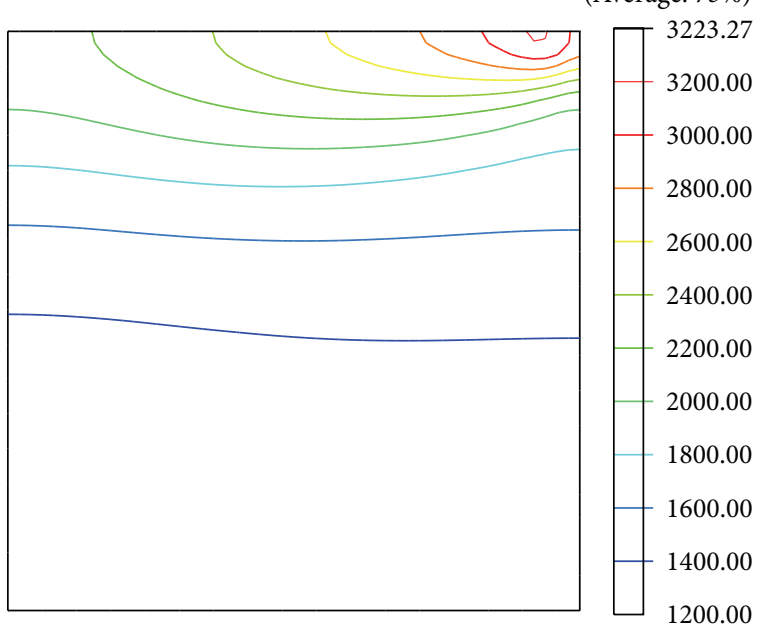

(a)

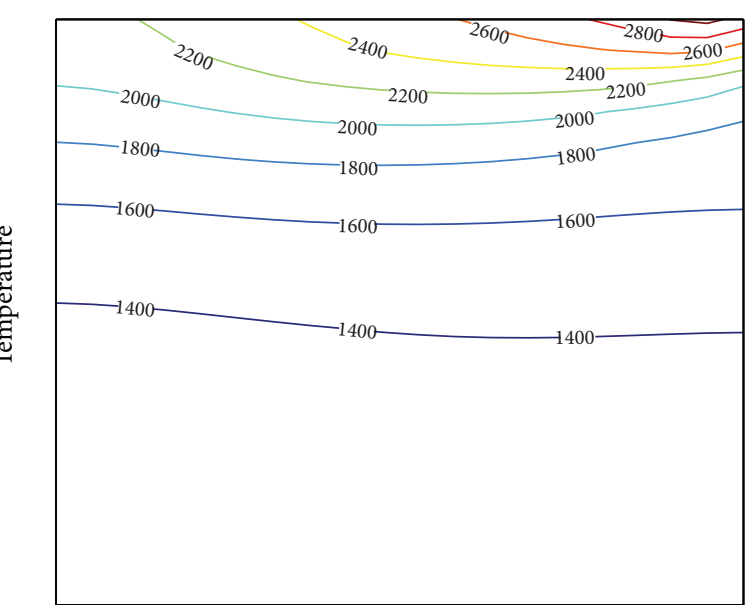

(b)

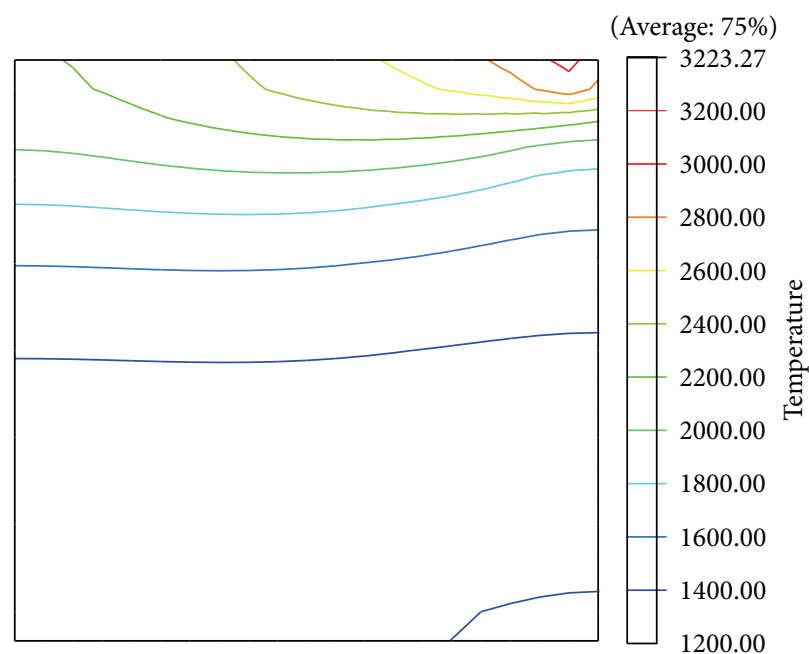

(c)

FIGURE 9: Temperature profile with 3D FE model (a), pseudo-analytical model on a coarse mesh (b), and coarse 3D FE model (c).

field within acceptable accuracy for most of the problem domain. The issue of inaccurate predictions at the boundaries has a greater effect on the results at the end of the parallel scanning strategy as the final scan track is quite close to the boundary. However, solutions to this problem have already been discussed in the previous section.

The characterization and zoning of the discrete domains/elements are shown in Figure 10 for all three models. It is again observed that the pseudo-analytical model has a better predictive capability than the coarse 3D FE model. Relative to the accurate 3D FEM, the coarse FE model is again found to have worse predictions than the pseudo-analytical model.

As in the previous case, the maximum domain temperature, the average domain temperature, and the standard deviation of temperature in the domain are tracked throughout the scan path and plotted through Figure 11 to Figure 13, respectively. In the parallel scanning strategy, the effect of change in direction on the temperature distribution is absent but the effect of boundaries is more prominent, as seen in the last section of Figure 11. However, the conservation and equivalence of energy in the models are still maintained, as seen in a similar average temperature prediction in Figure 12 and a correspondingly lower standard deviation of temperature at the end of scanning strategy in Figure 13. By considering the three history plots, it can be inferred that the coarse 3D FE model has a better spatial precision but a poorer temporal accuracy as compared to the pseudoanalytical model.

\section{Comparison of Scan Strategies}

Tables 2 and 3 show a comparison between the 3D FE models and pseudo-analytical method for the parallel and outspiral scan path, respectively. The values of each individual criterion and the dimensionless number $\theta$ were found to be 


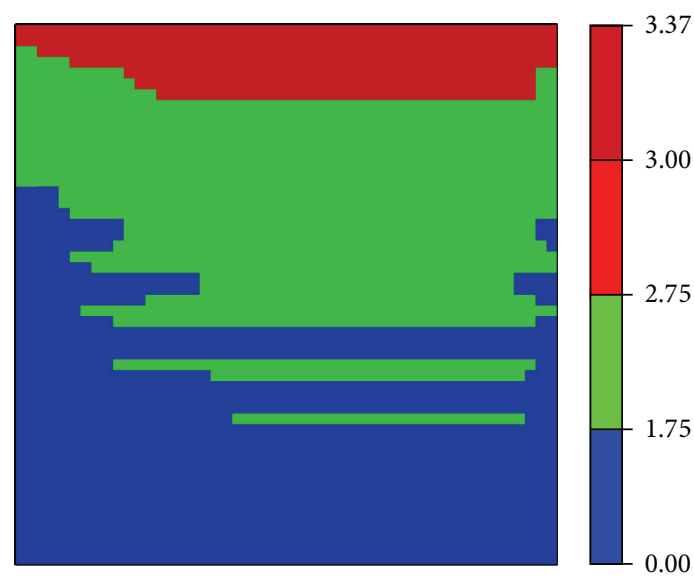

(a)

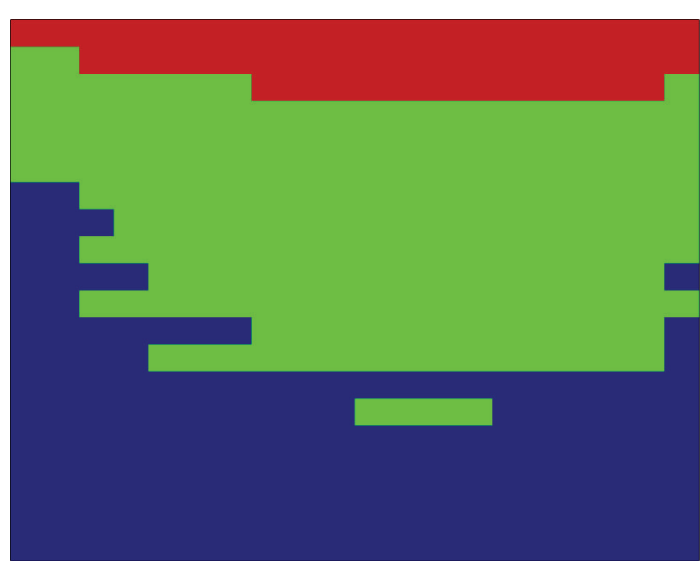

(b)

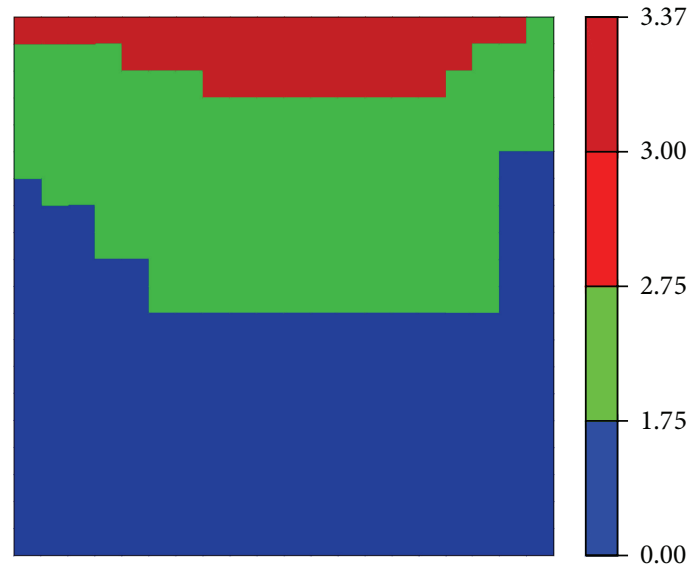

(c)

FIGURE 10: State of zones with 3D FE model (a) and pseudo-analytical model on a coarse mesh (b) and coarse 3D FE model (c) $(1=$ nonmolten (blue), 2 = molten (green), and 3 = overheated (red)).

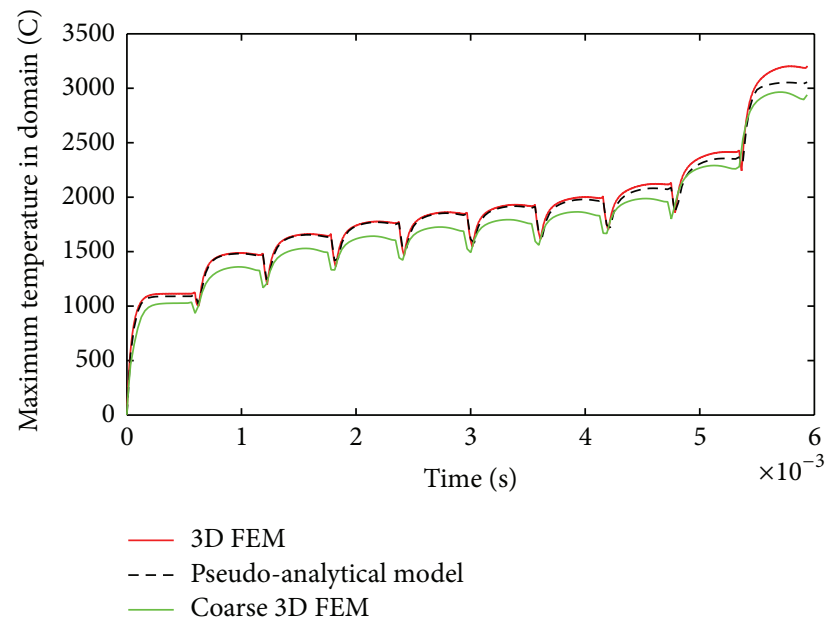

FIGURE 11: History plot for maximum temperature in modelled domain for parallel scan path as predicted with 3D FEM (red), pseudo-analytical model (black), and coarse 3D FEM (green). similar, if not the same. However the total time required for computation is much lower in the pseudo-analytical model than the 3D FE model.

The simulation time for the coarse 3D thermal FE model was found to be at par with the pseudo-analytical method. However, in the current simulations, the major calculation time consumption in the pseudo-analytical method was in the presolution stage, specifically during the characterization of the laser beam into a volumetric heat source, while the solution phase lasted for $\sim 4$ seconds in each case. In comparison, the solution phase for the coarse 3D FE model lasted for $\sim 18$ seconds. This time for computation of the heat source is an overhead and is only dependent on the relative size of the discrete domain with respect to the laser beam spot size. Thus, to properly ascertain the relative computational speed of the pseudo-analytical model over the 3D FEM, a scaling study was performed. The problem domain area was increased to twice, $4 \mathrm{x}$ and $16 \mathrm{x}$ times the problem area in the validation sections. The corresponding computational times 
TABLE 2: Comparison between 3D thermal FE models and pseudo-analytical model for parallel scan path.

\begin{tabular}{lccc}
\hline Features & 3D thermal FE model & Pseudo-analytical model & Coarse 3D thermal FE model \\
\hline Molten elements (\%) & 42.92 & 46.75 & 38.5 \\
Overheated elements $(\%)$ & 12.44 & 12.5 & 11 \\
Maximum Temperature $\left({ }^{\circ} \mathrm{C}\right)$ & 3200 & 3055.2 & 2964.87 \\
Maximum st. dev. of temperature $\left({ }^{\circ} \mathrm{C}\right)$ & 466.01 & 488.70 & 454.89 \\
Simulation time (s) & 5728.0 & 9.22 & 17.010 \\
$\theta$ & 1.9706 & 1.6959 & 1.8922 \\
\hline
\end{tabular}

TABLE 3: Comparison between 3D thermal FE models and pseudo-analytical model for out-spiral scan path.

\begin{tabular}{lccc}
\hline Features & 3D thermal FE model & Pseudo-analytical model & Coarse 3D thermal FE model \\
\hline Molten elements $(\%)$ & 45.08 & 43.5 & 44.75 \\
Overheated elements $(\%)$ & 9.32 & 12.75 & 2.5 \\
Maximum temperature $\left({ }^{\circ} \mathrm{C}\right)$ & 2878 & 2838.9 & 2587.76 \\
Maximum st. dev. of temperature $\left({ }^{\circ} \mathrm{C}\right)$ & 282.55 & 294.69 & 263.344 \\
Simulation time $(\mathrm{s})$ & 5896.5 & 9.37 & 18.22 \\
$\Theta$ & 1.9824 & 2.8625 & 0.3239 \\
\hline
\end{tabular}

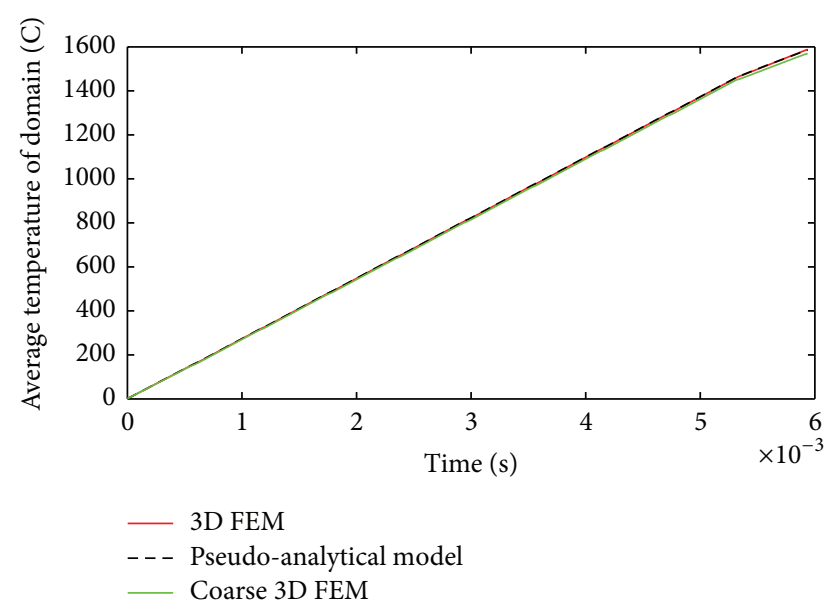

FIGURE 12: History plot for average domain temperature for parallel scan path as predicted with 3D FEM (red), pseudo-analytical model (black), and coarse 3D FEM (green).

were recorded for both the models and the result is shown in Figure 14.

\section{Discussions}

In the validation case studies, the pseudo-analytical model was thus found to be able to mimic the predictions of the corresponding high-fidelity finite element model. For each of the cases, it was also observed that the coarse FE model is incapable of accurate predictions, especially the zoning predictions. Simultaneously, the pseudo-analytical model

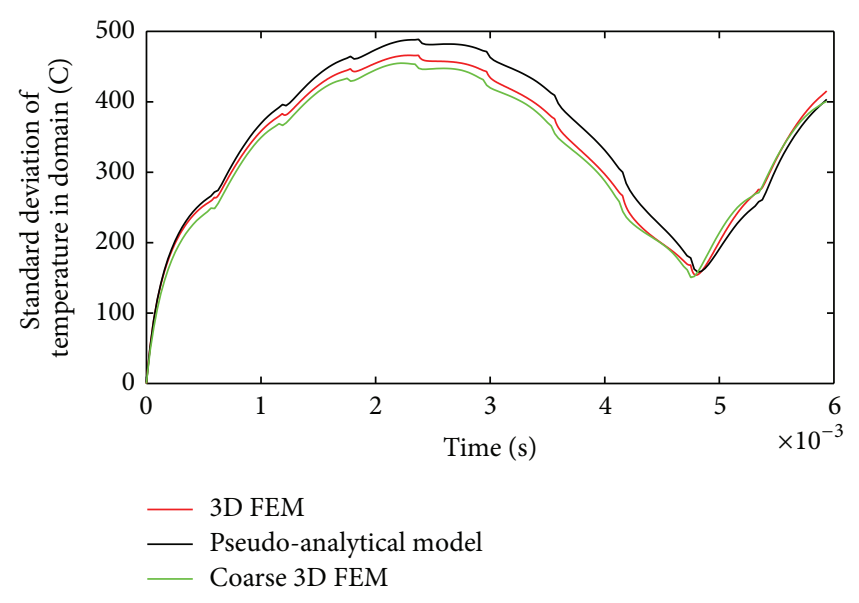

FIGURE 13: History plot for standard deviation of temperature in modelled domain as predicted with 3D FEM (red), pseudoanalytical model (black), and coarse 3D FEM (green).

was also found to have better domain scalability than both FE models. As the purpose of the research was to develop a fast yet reasonably accurate method that can be used for process optimization studies, the coarse FE model is found to be ineligible. Thus, in the following sections of the paper only the pseudo-analytical model is considered.

\section{Application in Optimized Cellular Scanning Strategy}

The greater speed and good scalability of the pseudoanalytical model, albeit at the cost of accuracy, make it suitable for optimization studies involving several model 


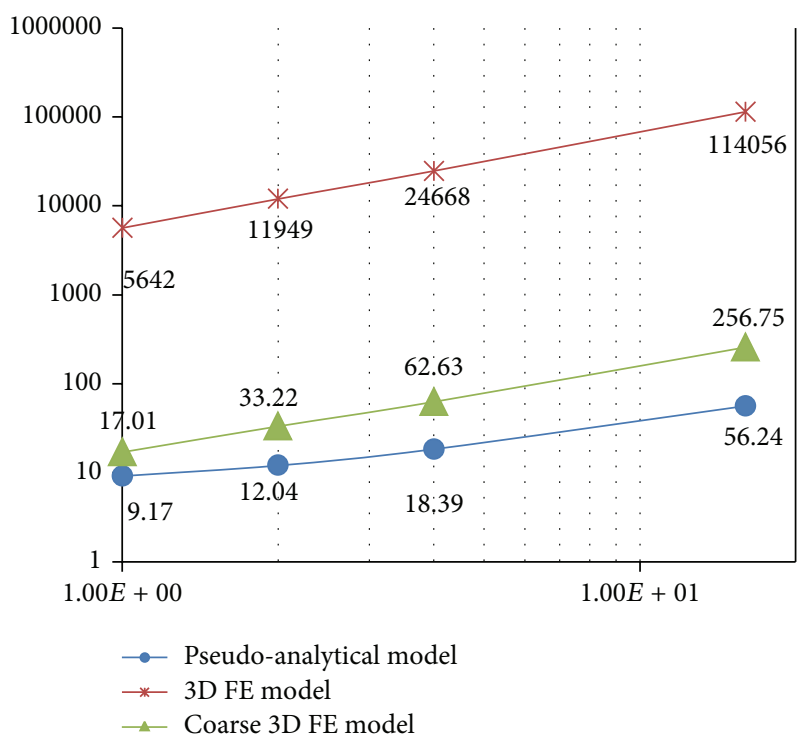

FIGURE 14: Domain scaling study for 3D FE models and pseudoanalytical method.

ฯ

(a)

\begin{tabular}{|c|c|c|c|}
\hline 13 & 14 & 15 & 16 \\
\hline 9 & 10 & 11 & 12 \\
\hline 5 & 6 & 7 & 8 \\
\hline 1 & 2 & 3 & 4 \\
\hline
\end{tabular}

(b)
FIGURE 15: Random processing sequence of unit cells for the cellular scanning strategy to be optimized (a) and linear processing sequence (b).

runs. As a test case, optimized cellular scanning strategies are generated for a sample domain of $2 \mathrm{~mm} \times 2 \mathrm{mmm} \times$ $50 \mu \mathrm{m}$. The sample is divided into 16 unit cells of $500 \mu \mathrm{m} \times$ $500 \mu \mathrm{m}$, which are to be processed in a particular sequence using one of the six scanning strategies shown in Figure 2. While it is acknowledged that to determine an optimized scanning strategy for the sample both the scanning strategy for each unit cell and the sequence of processing of the unit cells need to be determined, the latter is recognized to be a combinatorial optimization problem (similar to the travelling salesman problem) and is deemed to be beyond the scope of this paper. Instead a random sequence, shown in Figure 15, is generated for the processing of the unit cells in the sample domain and is adopted for the entire optimization study.

As the objective of the study is to show the potential of the pseudo-analytical method rather than the development of a better optimization algorithm, the global optimization toolbox available in the commercial software MATLAB is used. The single-objective genetic algorithm (ga) module is used for determining the optimum choice of scanning strategy for each unit cell. A population comprising of 24 individuals is evolved over 20 generations with an elite population of 2 individuals in each generation. The best fitness value and the mean fitness value of the population are tracked through the generations. Each individual is defined as a real array of 16 elements with the index of each element corresponding to the number of the unit cell for which the element denotes the scanning strategy. Each element is allowed to have any real value between $(-6,6]$ which is converted to an integer whole number, using a ceiling function, each of which corresponds to one of the scanning strategies. Thus, the genetic algorithm is used to scan a 16-dimensional space with 6 possible locations along each dimension.

Three different optimization simulations are carried out with different fitness criteria and the resulting scanning strategies are compared. The first optimization simulation has the standard deviation of temperature at the end of the process as the fitness criterion for minimization, thereby optimizing the scanning strategies for thermal homogeneity. The second optimization simulation considers the total consolidated area (comprising both molten and overheated zones) as the fitness criterion to be maximized, thereby optimizing for greater part density. For the third optimization simulation, the dimensionless number $\theta$ introduced earlier in this paper is utilized as a minimization criterion, thereby optimizing for the highest overall efficacy of the process. The three criteria focus on different output characteristics of the process and can be conceived to have an inherent paretooptimality with respect to each other (an aspect to be considered in successive studies). Additionally, two control test cases were simulated. In the first case, all the unit cells were to be processed with the parallel scanning strategy but in the same sequence as the optimization simulation. In the second case, the sequence of processing was also modified to a linear sequence following a left-to-right bottom-to-top approach (Figure 15).

The best fitness value and the mean fitness value are plotted against the generation number for all three optimization simulations (Figure 16). The genetic algorithm is shown to produce a convergent behaviour as the generations progress. This provides a proof of well-suitability of the genetic algorithm and the pseudo-analytical model for the optimization problem. Figure 17 shows the cellular scanning strategies obtained after 20 generations for the three independent optimization simulations. The five comparison criteria, as used in the model validation section, have been tabulated in Table 4 for the three optimized scanning strategies as well as the two control test cases.

The scanning strategies obtained at the end of the optimization simulations are indeed found to be better than the control test cases with respect to the criteria they have been optimized for. It is also observed that the choice of sequence has a pertinent effect on the comparison criteria, therefore, reaffirming that the generation of a properly optimized scanning strategy would also require the optimization of the processing sequence. 


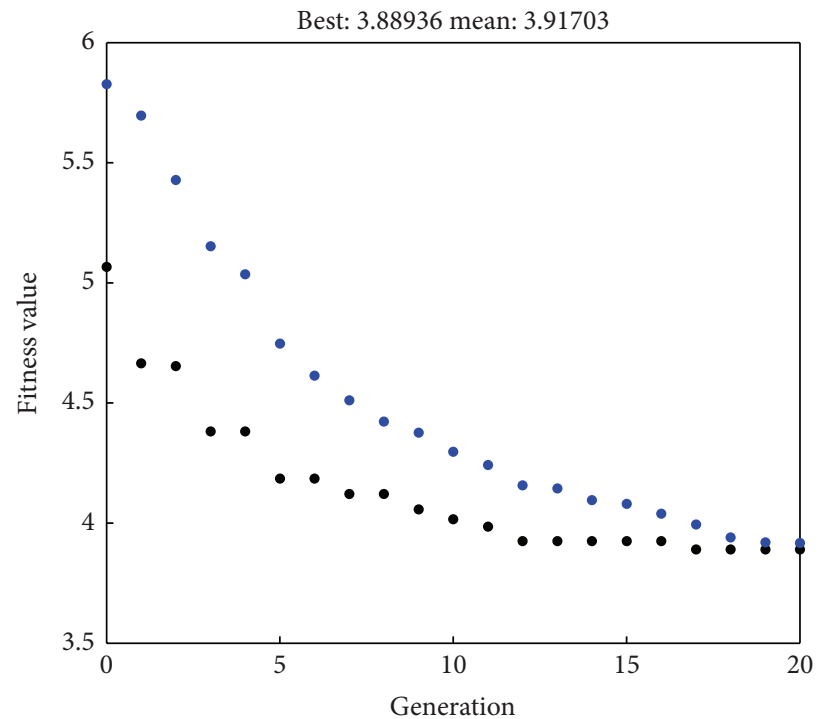

- Best fitness

- Mean fitness

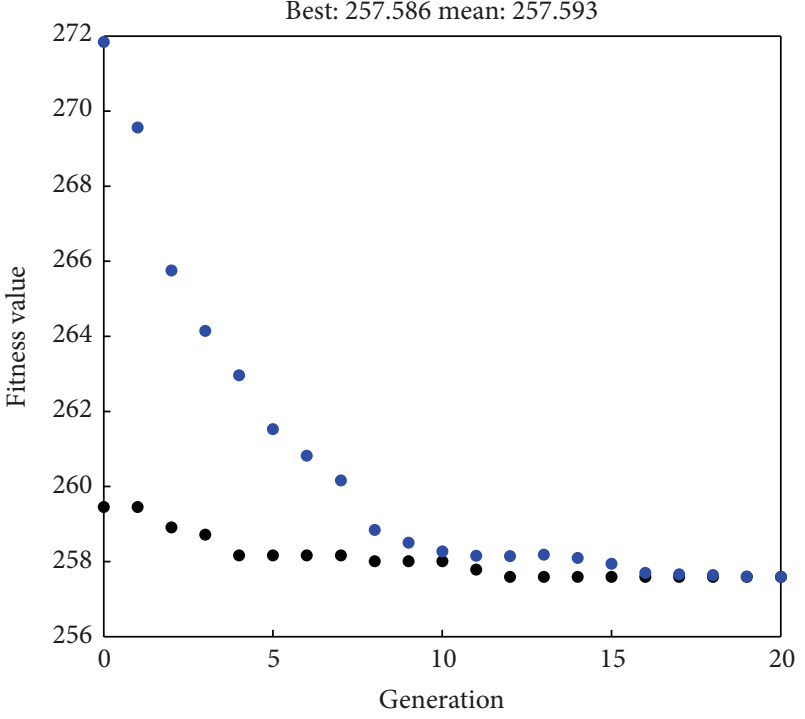

- Best fitness

- Mean fitness

(a)

(b)

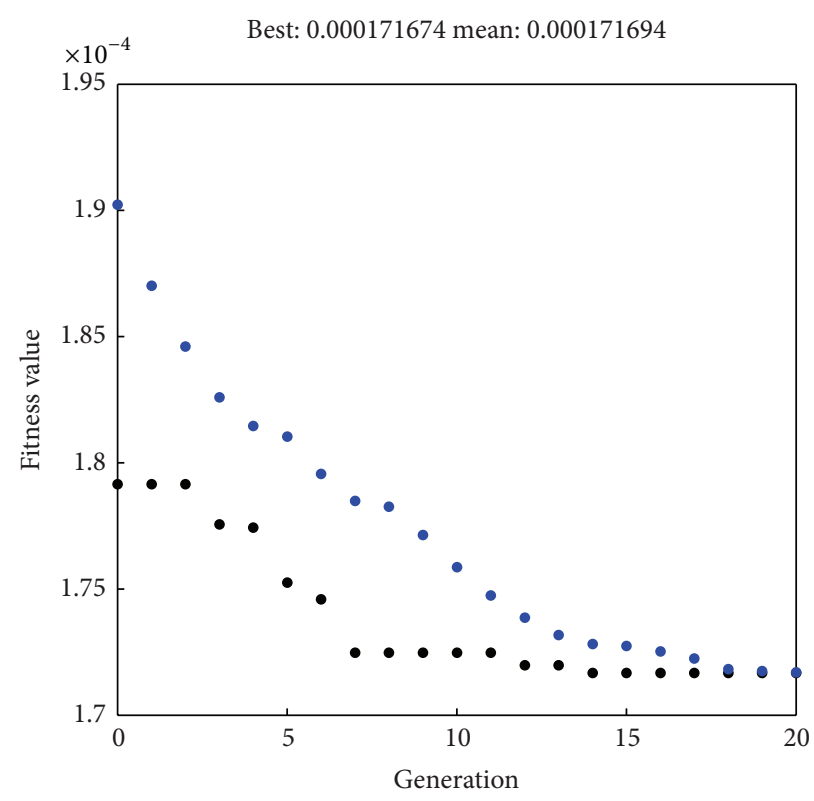

- Best fitness

- Mean fitness

(c)

Figure 16: Best and mean fitness value versus generation for optimization simulations with respect to $\theta$ (a), thermal homogeneity (b), and total consolidated area (c).

\section{Conclusion}

The primary ideas presented in the paper are summarized below.

(i) A fast, low-fidelity pseudo-analytical model has been proposed. The pseudo-analytical model has been validated through comparisons with results from $3 \mathrm{D}$ finite element models developed using the commercial software ABAQUS.

(ii) Compared to an accurate 3D FE model, the pseudoanalytical model was found to predict results with similar trends but at a computational time smaller by 2-3 orders of magnitude. 


\begin{tabular}{|c|c|c|c|}
\hline OS & AP & IS & PI \\
\hline P & IS & AP & P \\
\hline OS & OS & AP & AP \\
\hline AP & IS & IS & IS \\
\hline
\end{tabular}

(a)

\begin{tabular}{|c|c|c|c|}
\hline OS & AP & OS & AP \\
\hline AP & P & AP & AP \\
\hline OS & PIR & IS & AP \\
\hline AP & AP & PIR & P \\
\hline
\end{tabular}

(b)

\begin{tabular}{|c|c|c|c|}
\hline OS & OS & IS & P \\
\hline IS & AP & AP & AP \\
\hline OS & OS & AP & AP \\
\hline OS & OS & PIR & P \\
\hline
\end{tabular}

(c)

FIGURE 17: Cellular scanning strategy obtained from optimization simulations with respect to $\theta$ (a), thermal homogeneity (b), and total consolidated area (c) (IS = in-spiral, OS = out-spiral, AP- = antiparallel, P = parallel, $\mathrm{PI}=$ parallel interlaced, and PIR = parallel interlaced reverse).

TABLE 4: Comparison criteria for optimized scanning strategies and control test cases.

\begin{tabular}{lccccc}
\hline \multirow{2}{*}{ Features } & \multicolumn{2}{c}{ Optimized scanning strategies } & \multicolumn{3}{c}{ Control scanning strategy } \\
& Thermal homogeneity & Total consolidated area & $\theta$ & Case I & Case II \\
\hline Molten elements $(\%)$ & 55.60 & 56.21 & 59.93 & 48.37 & 47.06 \\
Overheated elements $(\%)$ & 33.29 & 34.79 & 27.89 & 35.79 & 36.82 \\
Maximum temperature $\left({ }^{\circ} \mathrm{C}\right)$ & 3200 & 3200 & 3200 & 3200 & 3200 \\
Maximum st. dev. of temperature $\left({ }^{\circ} \mathrm{C}\right)$ & 379.27 & 384.39 & 382.96 & 390.76 & 509.11 \\
Simulation time & $917 \mathrm{~s}$ & $916 \mathrm{~s}$ & $917 \mathrm{~s}$ & $916 \mathrm{~s}$ & $917 \mathrm{~s}$ \\
$\theta$ & 5.0527 & 5.1535 & 3.8894 & 6.0605 & 4.9191 \\
\hline
\end{tabular}

(iii) The pseudo-analytical model was found to have computation time similar to the corresponding 3D finite element model with similar element dimensions and time step size. This coarse FEM was observed to produce inaccurate results.

(iv) A small optimization study has been conducted using the pseudo-analytical model. Optimized scanning strategies were predicted for complete processing of a 16x larger domain (resulting in a 256x larger problem than the validation cases) based on three optimization criteria.

The low computational time, good scalability, and acceptable accuracy in prediction of certain criteria with respect to a 3D FE model provide a large potential for applicability of the pseudo-analytical model.

In the current paper, an effort has been made to select appropriate test cases which highlight the strengths as well as the shortcomings of the method. Thus, an ample scope of further improvement in the pseudo-analytical model has also been identified. The usage of two-dimensional analytical solutions in each discrete domain, the effects of nonlinear temperature dependency of material properties, the application of analytical element methods to better predict solutions at the boundary, and the corresponding calibrations of the pseudo-analytical model have been identified as potential areas and subsequent papers would deal with these issues. However, the focus of such development would still remain to construct a fast, reasonably accurate methodology rather than a high accuracy technique as such tools already exist in the form of finite element methods.

\section{Conflict of Interests}

The authors declare that there is no conflict of interests regarding the publication of this paper.

\section{References}

[1] K. Zeng, D. Pal, and B. Strucker, "A review of thermal analysi methods in Laser sintering and Selective laser melting," in Proceedings of the 23rd Annual International Solid Freeform Fabrication Symposium, Austin, Tex, USA, 2012.

[2] A. V. Gusarov, I. Yadroitsev, P. Bertrand, and I. Smurov, "Heat transfer modelling and stability analysis of selective laser melting," Applied Surface Science, vol. 254, no. 4, pp. 975-979, 2007.

[3] A. V. Gusarov, M. Pavlov, and I. Smurov, "Residual stresses at laser surface remelting and additive manufacturing," Physics Procedia, vol. 12, part A, pp. 248-254, 2011. 
[4] F. Gürtler, M. Karga, K. Leitza, and M. Schmidt, "Simulation of laser beam melting of steel powders using the threedimensional volume of fluid method," Physics Procedia, vol. 41, pp. 874-879, 2013.

[5] J. P. Kruth, L. Froyen, J. Van Vaerenbergh, P. Mercelis, M. Rombouts, and B. Lauwers, "Selective laser melting of ironbased powder," Journal of Materials Processing Technology, vol. 149, no. 1-3, pp. 616-622, 2004.

[6] F. Verhaeghe, T. Craeghs, J. Heulens, and L. Pandelaers, "A pragmatic model for selective laser melting with evaporation," Acta Materialia, vol. 57, no. 20, pp. 6006-6012, 2009.

[7] S. Koziel and L. Leifsson, Surrogate-Based Modeling and Optimization, Springer, New York, NY, USA, 2013. 


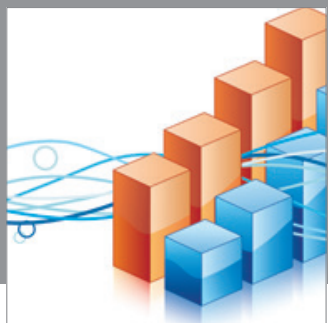

Advances in

Operations Research

mansans

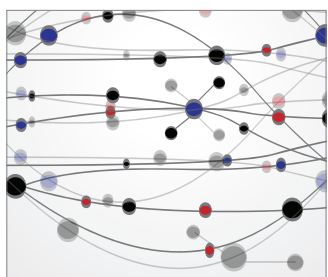

The Scientific World Journal
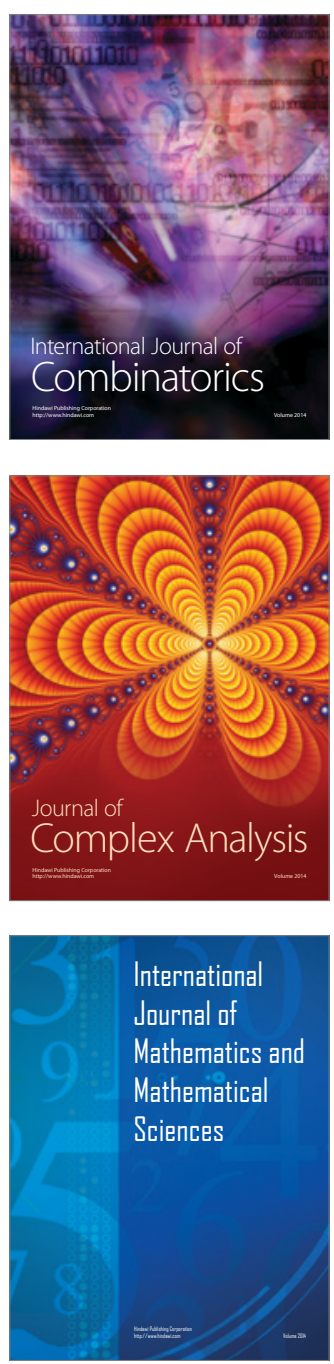
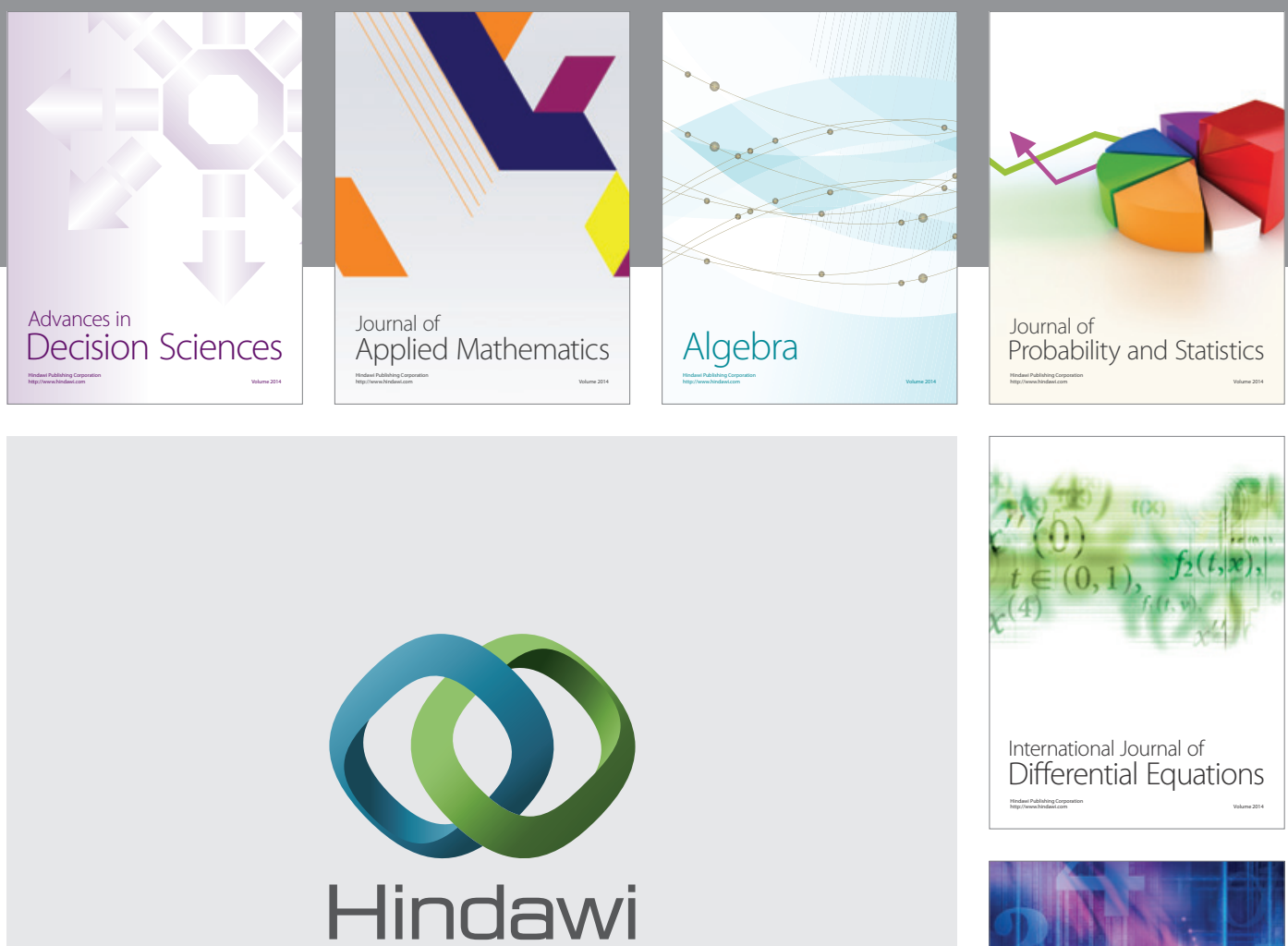

Submit your manuscripts at http://www.hindawi.com
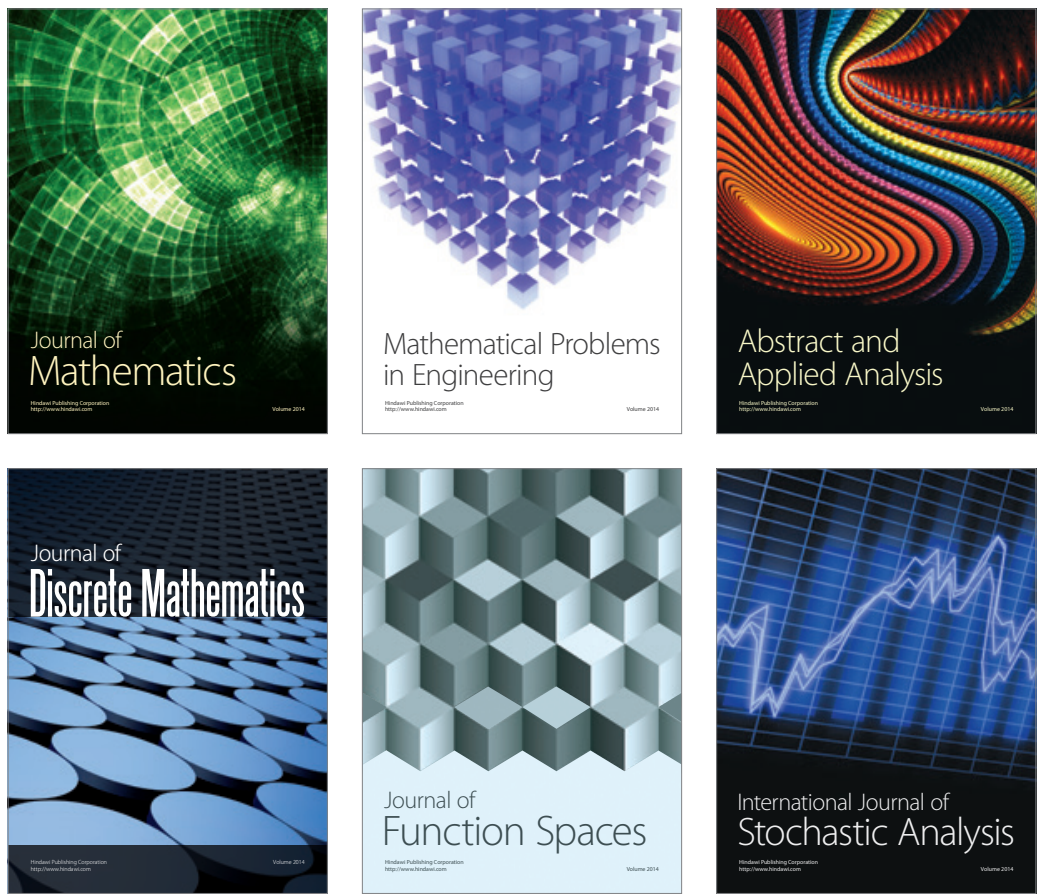

Journal of

Function Spaces

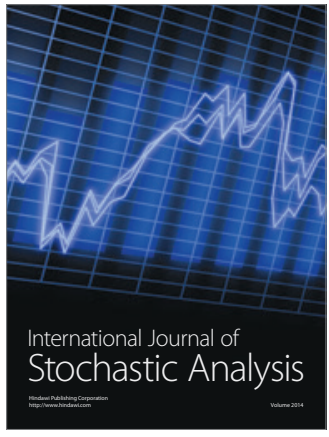

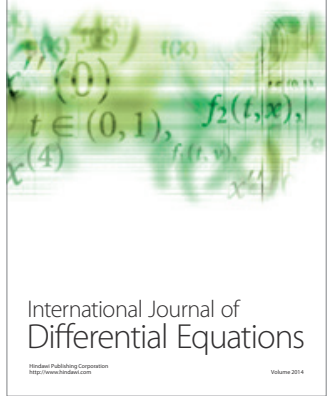
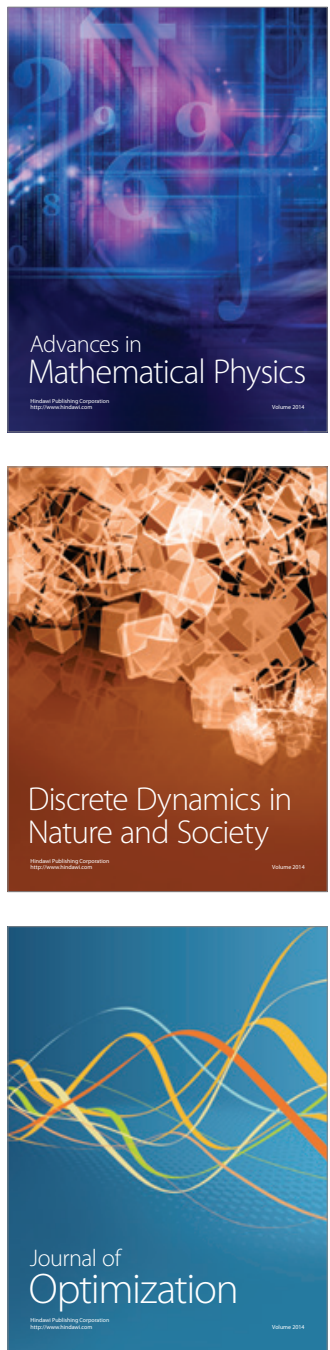